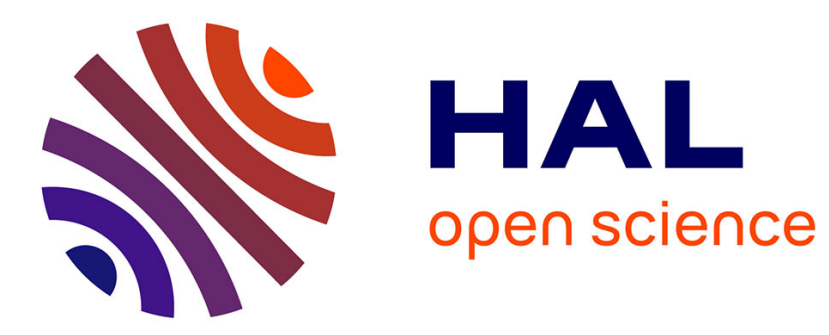

\title{
Excitation injector in an atomic chain: long-ranged transport and efficiency amplification
}

Pierre Doyeux, Riccardo Messina, Bruno Leggio, Mauro Antezza

\section{To cite this version:}

Pierre Doyeux, Riccardo Messina, Bruno Leggio, Mauro Antezza. Excitation injector in an atomic chain: long-ranged transport and efficiency amplification. Physical Review A : Atomic, molecular, and optical physics [1990-2015], 2017, 95 (95), pp.012138. 10.1103/PhysRevA.95.012138 . hal-01448442

\section{HAL Id: hal-01448442 \\ https://hal.science/hal-01448442}

Submitted on 13 Oct 2020

HAL is a multi-disciplinary open access archive for the deposit and dissemination of scientific research documents, whether they are published or not. The documents may come from teaching and research institutions in France or abroad, or from public or private research centers.
L'archive ouverte pluridisciplinaire HAL, est destinée au dépôt et à la diffusion de documents scientifiques de niveau recherche, publiés ou non, émanant des établissements d'enseignement et de recherche français ou étrangers, des laboratoires publics ou privés. 


\title{
Excitation injector in an atomic chain: Long-range transport and efficiency amplification
}

\author{
Pierre Doyeux, ${ }^{1}$ Riccardo Messina, ${ }^{1}$ Bruno Leggio,,${ }^{1, *}$ and Mauro Antezza ${ }^{1,2}$ \\ ${ }^{1}$ Laboratoire Charles Coulomb (L2C), UMR 5221 CNRS-Université de Montpellier, F- 34095 Montpellier, France \\ ${ }^{2}$ Institut Universitaire de France, 1 rue Descartes, F-75231 Paris, France \\ (Received 23 September 2016; published 27 January 2017)
}

\begin{abstract}
We investigate the transport of energy in a linear chain of two-level quantum emitters (atoms) weakly coupled to a blackbody radiation bath. We show that simply by displacing one or more atoms from their regular-chain positions, the efficiency of the energy transport can be considerably amplified of at least one order of magnitude. In addition, in configurations providing an efficiency greater than $100 \%$, the distance between the last two atoms of the chain can be up to 20 times larger than the one in the regular chain, thus achieving a much longer-range energy transport. By performing both a stationary and time-dependent analysis, we ascribe this effect to an elementary block of three atoms, playing the role of excitation injector from the blackbody bath to the extraction site. By considering chains with up to seven atoms, we also show that the amplification is robust and can be further enhanced up to $1400 \%$.
\end{abstract}

DOI: 10.1103/PhysRevA.95.012138

\section{INTRODUCTION}

Energy transport in quantum systems is a field of research that has recently received a lot of attention [1-3]. The possibility of transporting energy efficiently in quantum systems is not only of fundamental interest, but also promises many potential technological applications, e.g., in quantum thermodynamics [4,5]. As a matter of fact, it already has proven to be useful in numerous fields such as organic photovoltaic cells [6], quantum information [7], and, more generally, nanoscale technologies [1]. In addition, the experimental observations of light-harvesting proteins, such as the Fenna-Matthews-Olson (FMO) complex [8], have also triggered a lot of interest [9-12]. All of these systems require energy to be transported within quantum systems over distances that might be relatively large, and with as little losses as possible. A deeper comprehension of the mechanism at the origin of energy transport in such systems is therefore essential. To this end, the investigation of systems composed of two-level quantum emitters provides an ideal framework $[2,10,13-15]$.

One of the most challenging tasks remains to fully understand the role played by the environment in energy-transport processes. This role has been investigated both in weak and strong coupling in many aspects [16]: quantum systems inside in a cavity $[12,17]$ or placed close to metallic surfaces, such as a mirror [18] or nanospheres [19]. The boundary conditions at the edges of linear systems have also been studied [20] as well as the activation of nonlocal effects stemming from the environmental thermal bath [21].

In this latter work, the system under investigation is composed of a few two-level emitters embedded in blackbody radiation. The efficiency of the transport of incoherently pumped excitations is studied with respect to the spatial distribution of the emitters. In particular, two-dimensional (2D) and 3D configurations have been explored with the intention of mimicking the geometry of an FMO complex. Remarkably, in some cases, this efficiency can surpass the value of $100 \%$ and even reach $300 \%$, allowing one to extract

\footnotetext{
*Current address: Inria project Virtual Plants, CIRAD and INRA, F-34095 Montpellier, France.
}

three times more energy than the one pumped in. This is due to absorption of excitations during the transport process, which is triggered by nonlocal effects stemming from the presence of the thermal bath.

In this paper, we focus instead on the energy transport within a 1D system, which is a linear chain of a few twolevel quantum emitters (from now on referred to as atoms) weakly interacting with blackbody radiation at an arbitrary temperature. Similarly to [21], the bath correlations provide the atoms with the possibility to (collectively) absorb excitations from the reservoir and to store them within the atomic system. We show that for specific geometrical configurations of the chain, atomic triplets can form an elementary block acting as an excitation injector, resulting in a remarkable enhancement of one order of magnitude of the efficiency. In other words, this latter can reach values greater that $1000 \%$. In addition, these configurations realize energy transport over distances that are much larger than the one of the chain with regularly distributed atoms, which makes them particularly interesting for potential technological applications.

The paper is structured as follow: in Sec. II, we introduce the model and the quantities used to perform the study of energy transport in our system. We present the main results of the paper in Sec. III, i.e., the occurrence of efficiency amplification and long-range energy transport. Section IV is dedicated to the investigation, both at stationarity and during the dynamics, of the mechanisms producing these effects for a specific fouratom chain. Finally, in Sec. V, we analyze the robustness of efficiency amplification and long-range energy transport with respect to parameters such as, e.g., the electromagnetic (EM) bath temperature, the atomic frequency, etc. We also study these effects with linear chains composed of $N=5,6,7$ atoms.

\section{PHYSICAL SYSTEM}

In this section, we provide all the details of the formalism we use in the rest of the paper, so that the results of this paper could be easily followed and reproduced without ambiguity in the definition of the physical quantities.

The open system under investigation, depicted in Fig. 1, is a linear chain of $N$ identical two-level atoms of frequency $\omega$ which is embedded in an EM blackbody bath at temperature 


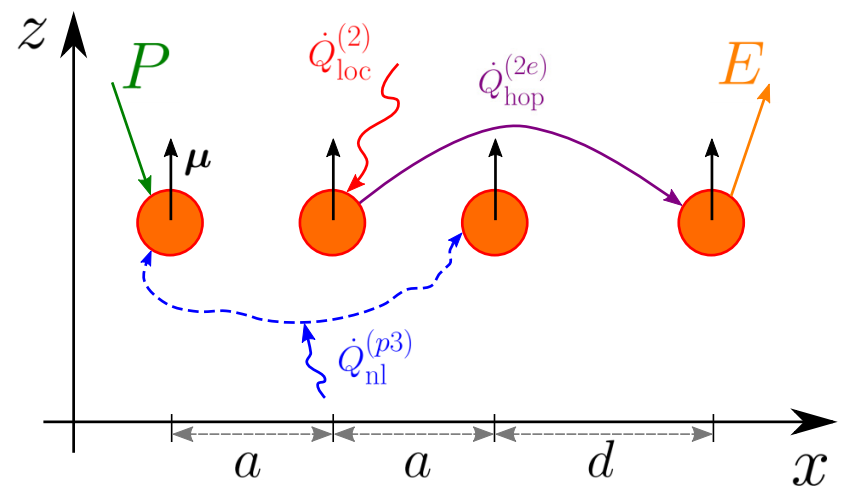

FIG. 1. Linear chain of four atoms with induced dielectric dipole $\boldsymbol{\mu}$ (black arrows) embedded in a thermal bath at $T$. Neighboring atoms are separated by a distance $a$, except the last two whose distance is $d$. Energy is pumped (extracted) within (from) the system with flux $P$ $(E)$ applied on the first (last) atom of the chain. An example of each type of heat flux (local, nonlocal, and hopping) has been represented in the figure.

$T$. In order to investigate energy-transport efficiency along the chain, excitations are incoherently pumped into the system on one edge (the pumping site $p$ ), while extraction is performed on the other one (the extraction site $e$ ). The atoms composing the chain are thus labeled as $\{p, 2, \ldots, N-1, e\}$, and we introduce the Cartesian coordinates $\{x, y, z\}$ where the $x$ axis is defined as the one on which the atoms are aligned.

Following [21], the total Hamiltonian describing this system is

$$
H_{\text {tot }}=H_{\mathrm{a}}+H_{\mathrm{B}}+H_{\mathrm{int}},
$$

where $H_{\mathrm{a}}$ and $H_{\mathrm{B}}$ are, respectively, the atomic and bath free Hamiltonians, and $H_{\text {int }}$ is the interaction Hamiltonian. More precisely, defining $\left|g_{j}\right\rangle\left(\left|e_{j}\right\rangle\right)$ the ground (excited) state of the $j$ th atom, and $\sigma_{j}^{-}=\left|g_{j}\right\rangle\left\langle e_{j}\right|\left(\sigma_{j}^{+}=\left|e_{j}\right\rangle\left\langle g_{j}\right|\right)$ the corresponding lowering (raising) operator, we have $H_{\mathrm{a}}=$ $\hbar \omega \sum_{j=1}^{N} \sigma_{j}^{+} \sigma_{j}^{-}$. In addition, assuming that each atom has the same dielectric dipole moment operator $\boldsymbol{\mu}$ and within the dipole approximation, the atom-bath interaction is given by $H_{\text {int }}=-\sum_{j=1}^{N} \boldsymbol{\mu} \cdot \mathbf{E}\left(\mathbf{r}_{j}\right)$, where $\mathbf{E}\left(\mathbf{r}_{j}\right)$ is the electric field at the position $\mathbf{r}_{j}$ of the $j$ th atom.

\section{A. Master equation}

Within the weak-coupling limit, the Born-Markov approximation [22] allows us to describe the time evolution of the reduced atomic density matrix $\rho(t)$ with the following quantum Markovian master equation [21,23]:

$$
\dot{\rho}=-\frac{i}{\hbar}\left[H_{\mathrm{sys}}, \rho\right]+D_{\mathrm{loc}}[\rho]+D_{\mathrm{nl}}[\rho]+D_{\mathrm{in}}[\rho]+D_{\text {out }}[\rho],
$$

where here and in the following the explicit time dependence of $\rho$ and $\rho$-dependent quantities will be dropped for simplicity. The effective Hamiltonian reads $H_{\text {sys }}=H_{\mathrm{a}}+H_{\Lambda}$, where

$$
H_{\Lambda}=\sum_{j<k}^{N} H_{\Lambda}^{(j k)}=\hbar \sum_{j<k}^{N} \Lambda_{j k}\left(\sigma_{j}^{+} \sigma_{k}^{-}+\sigma_{k}^{+} \sigma_{j}^{-}\right)
$$

characterizes the unitary evolution of the atomic system. The coefficient $\Lambda_{j k}$ is the interaction strength between induced dipoles of the transitions of atoms $j$ and $k$, which is mediated by the EM field, and is given by

$$
\begin{aligned}
\Lambda_{j k} & =-\frac{3}{4} \gamma_{0}\left\{2\left(\hat{\boldsymbol{\mu}} \cdot \hat{\mathbf{r}}_{j k}\right)^{2} f\left(\tilde{r}_{j k}\right)+\left[1-\left(\hat{\boldsymbol{\mu}} \cdot \hat{\mathbf{r}}_{j k}\right)^{2}\right] g\left(\tilde{r}_{j k}\right)\right\} \\
f(x) & =\frac{\cos x+x \sin x}{x^{3}}, \quad g(x)=\frac{\left(x^{2}-1\right) \cos x-x \sin x}{x^{3}},
\end{aligned}
$$

where the coefficient $\gamma_{0}=|\boldsymbol{\mu}|^{2} \omega^{3} / 3 \pi \epsilon_{0} \hbar c^{3}$ is the rate of spontaneous emission in vacuum. We have also introduced $\hat{\boldsymbol{\mu}}=\frac{\boldsymbol{\mu}}{|\boldsymbol{\mu}|}, \hat{\mathbf{r}}_{j k}=\frac{\mathbf{r}_{j k}}{\left|\mathbf{r}_{j k}\right|}$, where $\mathbf{r}_{j k}=\mathbf{r}_{j}-\mathbf{r}_{k}$ and $\tilde{r}_{j k}=\frac{\omega}{c}\left|\mathbf{r}_{j k}\right|$. It is worth stressing that $\Lambda_{j k}$ dramatically increases when the distance between the two atoms is smaller than $c / \omega$.

The nonunitary interaction between the atomic system and the bath is described by the so-called dissipators. First, each atomic transition exchanges excitations with the thermal bath by emitting or absorbing a photon of frequency $\omega$. The local dissipators capture these interactions,

$$
\begin{aligned}
D_{\mathrm{loc}}[\rho]= & \sum_{j} D_{\mathrm{loc}}^{(j)}[\rho] \\
= & \sum_{j}\left\{n \gamma_{0}\left(\sigma_{j}^{+} \rho \sigma_{j}^{-}-\frac{1}{2}\left\{\sigma_{j}^{-} \sigma_{j}^{+}, \rho\right\}\right)\right. \\
& \left.+(1+n) \gamma_{0}\left(\sigma_{j}^{-} \rho \sigma_{j}^{+}-\frac{1}{2}\left\{\sigma_{j}^{+} \sigma_{j}^{-}, \rho\right\}\right)\right\},
\end{aligned}
$$

where $n=\left[\exp \left(\hbar \omega / k_{B} T\right)-1\right]^{-1}$ is the average number of photons with energy $\hbar \omega$ when the bath is at temperature $T$ and with $\{\cdot, \cdot\}$ denoting an anticommutator.

In addition to a local dissipator, involving atoms separately, we should also take into account that each pair of atoms may interact with the EM field with a collective coherent behavior: the two atoms behave as a single entity, and they trade the same amount of energy with the environmental bath, both of them absorbing or emitting energy in a unique process. This nonlocal dissipation phenomenon is triggered by the autocorrelation functions of the EM field which allow resonant atomic transitions to cooperate. The corresponding nonlocal dissipator reads

$$
\begin{aligned}
D_{\mathrm{nl}}[\rho]= & \sum_{j<k} D_{\mathrm{nl}}^{(j k)}[\rho] \\
= & \sum_{j<k}\left\{n \gamma_{j k}\left(\sigma_{j}^{+} \rho \sigma_{k}^{-}-\frac{1}{2}\left\{\sigma_{k}^{-} \sigma_{j}^{+}, \rho\right\}\right)\right. \\
& \left.+(1+n) \gamma_{j k}\left(\sigma_{j}^{-} \rho \sigma_{k}^{+}-\frac{1}{2}\left\{\sigma_{k}^{+} \sigma_{j}^{-}, \rho\right\}\right)+\text { H.c. }\right\},
\end{aligned}
$$

where $\gamma_{j k}$ is the rate of spontaneous emission of the atomic pair $(j, k)$ which is obtained as

$$
\gamma_{j k}=\gamma_{0} \sum_{l=1,2,3}\left([\hat{\boldsymbol{\mu}}]_{l}\right)^{2} \alpha_{j k}^{(l)}
$$


with $l$ denoting the three Cartesian components, and where we used

$$
\begin{gathered}
\alpha_{j k}^{(1)}=\frac{3}{\tilde{r}_{j k}^{3}}\left(\sin \tilde{r}_{j k}-\tilde{r}_{j k} \cos \tilde{r}_{j k}\right), \\
\alpha_{j k}^{(2)}=\alpha_{j k}^{(3)}=\frac{3}{2 \tilde{r}_{j k}^{3}}\left[\tilde{r}_{j k} \cos \tilde{r}_{j k}+\left(\tilde{r}_{j k}^{2}-1\right) \sin \tilde{r}_{j k}\right] .
\end{gathered}
$$

Under the sole action of the local and nonlocal dissipators, the system would reach thermal equilibrium with the bath at temperature $T$. However, the presence of incoherent pumping and extraction perturbs the system, preventing it from reaching its Gibbs state. The pumping performed on $p$ is described by

$$
D_{\text {in }}[\rho]=\gamma_{\text {in }}\left(\sigma_{p}^{+} \rho \sigma_{p}^{-}-\frac{1}{2}\left\{\sigma_{p}^{-} \sigma_{p}^{+}, \rho\right\}\right),
$$

where $\gamma_{\text {in }}$ is the pumping rate. The dissipator corresponding to the extraction occurring on $e$ with rate $\gamma_{\text {out }}$ is

$$
D_{\text {out }}[\rho]=\gamma_{\text {out }}\left(\sigma_{e}^{-} \rho \sigma_{e}^{+}-\frac{1}{2}\left\{\sigma_{e}^{+} \sigma_{e}^{-}, \rho\right\}\right) .
$$

\section{B. Heat fluxes and efficiency}

In order to understand the energy-transport properties of our system, it is natural to identify the different channels through which the atomic system can either absorb, emit, or transmit energy. Before entering into detail, let us precisely state that the energy exchanges occurring in our system are heat fluxes as defined in the framework of quantum thermodynamics [24,25]. No work is involved since $\partial H_{\text {sys }} / \partial t=0$.

Let us start with the so-called hopping heat fluxes. These occur between two atomic resonant transitions and represent energy exchanged between two atoms without modifying the energy content of the environment. Therefore, these fluxes do not change the energy of the global atomic system, but rather describe trades of excitations between its different subparts. More specifically, they stem from the field-induced dipoledipole interactions and, for the atom $j$, are defined as

$$
\dot{Q}_{\text {hop }}^{(j k)}=-\frac{i}{\hbar} \operatorname{Tr}\left(H_{\mathrm{a}}^{(j)}\left[H_{\Lambda}^{(j k)}, \rho\right]\right),
$$

where $j$ and $k(j \neq k)$ index two atoms and with $H_{\mathrm{a}}^{(j)}=$ $\hbar \omega \sigma_{j}^{+} \sigma_{j}$. With this definition, having $\dot{Q}_{\text {hop }}^{(j k)}>0$ means that atom $j$ is absorbing energy at the expense of $k$. Moreover, note that $\dot{Q}_{\text {hop }}^{(k j)}=-\dot{Q}_{\text {hop }}^{(j k)}$. It is worth stressing that Eq. (12) can be rewritten, after straightforward calculations, as

$$
\dot{Q}_{\mathrm{hop}}^{(j k)}=-2 \hbar \omega \Lambda_{j k} \operatorname{Im}\left(c^{(j k)}\right),
$$

where $c^{(j k)}=\left\langle\sigma_{j}^{+} \sigma_{k}^{-}\right\rangle$is the coherence between atoms $j$ and $k$. Equation (13) encapsulates two essential features of the hopping fluxes. First, they depend on the amplitude of the dipole-dipole interaction $\Lambda_{j k}$, notably inheriting its spatial dependence. Second, the existence and the strength of the hopping fluxes directly depend on the presence of nonreal coherences between the two atoms. Note that this term of energy hopping between sites is often the only one considered in standard transport models allowing energy to be transported along atomic systems $[12,17]$.

We now turn to the fluxes stemming from the dissipators, starting with the local ones. They describe the exchanges of excitations between an atomic transition and the modes of the EM bath at the same frequency,

$$
\dot{Q}_{\mathrm{loc}}^{(j)}=\operatorname{Tr}\left(H_{\mathrm{a}} D_{\mathrm{loc}}^{(j)}[\rho]\right),
$$

where $\dot{Q}_{\text {loc }}^{(j)}>0(<0)$ means that the transition is absorbing (emitting) energy from (to) the environment.

Similarly, the nonlocal dissipators produce heat fluxes between an atomic pair and the modes of the environmental field. Focusing, for example, on the couple $(j, k)$, the energy absorbed (emitted) by atom $j$ reads

$$
\dot{Q}_{\mathrm{nl}}^{(j k)}=\operatorname{Tr}\left(H_{a}^{(j)} D_{\mathrm{nl}}^{(j k)}[\rho]\right) .
$$

In the case $\dot{Q}_{\mathrm{nl}}^{(j k)}>0(<0)$, the atoms $j$ and $k$ both absorb (emit) an equal amount of energy $\dot{Q}_{\mathrm{nl}}^{(j k)}$ from (to) the EM field. Straightforward calculations lead to the following expression of the nonlocal heat fluxes:

$$
\dot{Q}_{\mathrm{nl}}^{(j k)}=-\hbar \omega \gamma_{j k} \operatorname{Re}\left(c^{(j k)}\right) .
$$

Thus, similarly to the hopping fluxes, the nonlocal ones can only exist in the presence of coherences. It is precisely the presence of these terms, usually neglected in models of quantum energy transport, that triggers remarkable effects on the transport efficiency, as will be clarified in the next sections.

Finally, the heat flux of excitations injected into $p$ is

$$
P=\operatorname{Tr}\left(H_{\mathrm{a}} D_{\text {in }}[\rho]\right) \text {. }
$$

Note that $P \geqslant 0$ since the energy of the atomic system is always increased by this flux.

On the other hand, the extraction lowers the energy of the atomic system. Thus, in order to have a positive quantity, we define

$$
E=-\operatorname{Tr}\left(H_{\mathrm{a}} D_{\text {out }}[\rho]\right)
$$

which corresponds to the energy extracted from $e$, so that $E \geqslant 0$.

Following [21], we use the quantities introduced in Eqs. (17) and (18) to investigate the energy-transport efficiency along the chain, which we define as

$$
\chi=\frac{E-E_{0}}{P},
$$

where $E_{0}$ is the field thermal energy extracted from $e$ in the absence of pumping, i.e., in the limit $\gamma_{\text {in }} \rightarrow 0$. Note that although $\gamma_{\text {in }}$ does not explicitly appear in Eq. (18), $E$ is a function of $\gamma_{\text {in }}$ due to the dependence of the atomic state $\rho$ on the pumping rate.

In particular, having $\chi=1$ means that the amount of energy pumped into $p$ equals the extra energy extracted in the presence of pumping, suggesting that the excitations have been transported throughout the chain without any loss or gain. In general, $\chi$ is a function of time because of the $t$ dependence of $\rho$. In what follows, we will study both the stationary efficiency $\chi_{\text {stat }}$, obtained in the limit $t \rightarrow \infty$, and the dynamic behavior of the efficiency. 


\section{EFFICIENCY AMPLIFICATION AND LONG-RANGE TRANSPORT}

We dedicate this section to the presentation of the main result of this paper, namely, the amplification of long-range energy-transport efficiency, through the specific example of the four-atom chain represented in Fig. 1.

Before getting to the heart of the matter, here we set some parameters that will be used for the simulations throughout this paper. The regular configuration that we take as a reference has $d=a=0.1 \mu \mathrm{m}$. The dipoles are oriented along the $z$ direction, i.e., orthogonally to the chain, with magnitude $|\boldsymbol{\mu}|=10^{-30} \mathrm{Cm}$. The rates of pumping and extraction are $\gamma_{\text {in }}=10^{-3} \gamma_{0}$ and $\gamma_{\text {out }}=10^{2} \gamma_{0}$. These values have been used, for instance, in [21] to simulate models analogous to FMO complexes. Note that $\gamma_{\text {in(out) }}$ depends on the frequency through $\gamma_{0}$. The numerical results have been obtained using the opensource package QuTiP [26,27].

Let us now introduce the effects of efficiency amplification and long-range transport in the case of a four-atom chain $\{p, 2,3, e\}$ with an atomic frequency of $\omega=1 \times 10^{14} \mathrm{rad} \mathrm{s}^{-1}$. The issue we address here is the robustness of energy-transport efficiency at stationarity with respect to the displacement of the extraction site, the last atom of the chain in our case, and the temperature of the EM bath. As we will see, these two parameters play a crucial role in the efficiency amplification mechanism. To this end, we plot in Fig. 2 the efficiency as atom $e$ is moved away from the rest of the chain. This has been simulated for two temperatures: $T=10 \mathrm{~K}$ to reproduce an environment close to absolute zero and $T=300 \mathrm{~K}$, the scenario at ambient temperature being experimentally relevant as well.

At $T=10 \mathrm{~K}$ (green curve), the efficiency reaches at most $100 \%$ and then decreases monotonically with $d$, the maximum of efficiency occurring in the regular configuration $(d=a)$.

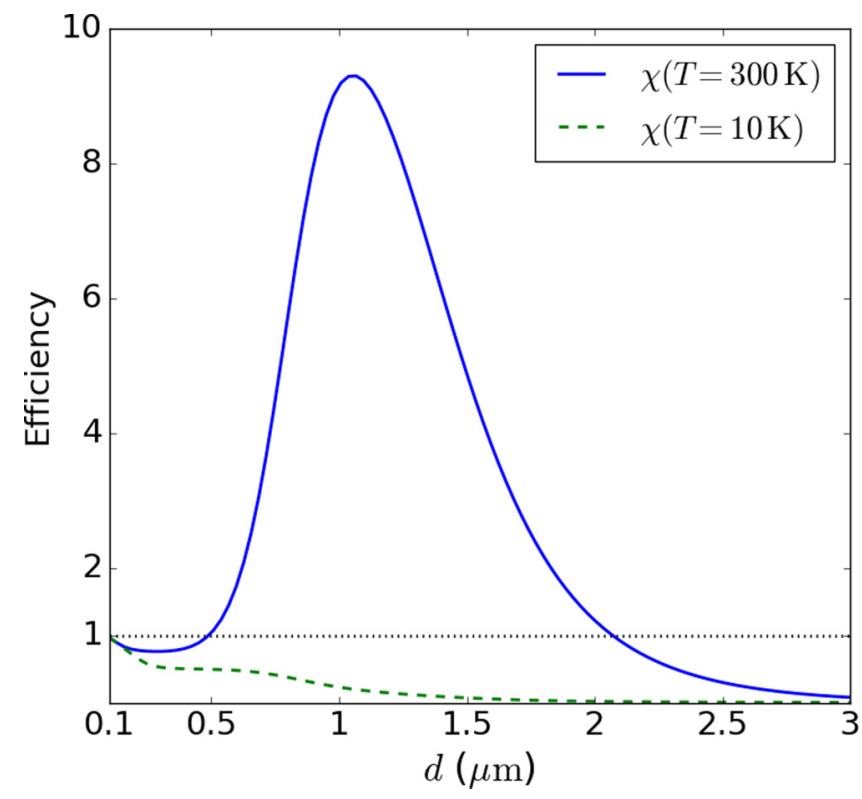

FIG. 2. Efficiency as a function of the distance between atom $e$ and the rest of the chain at $T=10 \mathrm{~K}$ (dashed green line) and $T=300 \mathrm{~K}$ (solid blue line).
On the contrary, when the displacement is realized at $T=$ $300 \mathrm{~K}$ (blue curve), the efficiency is remarkably amplified up to $\simeq 900 \%$. Besides, we have $\chi \geqslant 100 \%$ for a wide range of $d>a$, meaning that when the efficiency is amplified, the energy transport is performed over larger distances than in the case $T=10 \mathrm{~K}$

To gain more insight into the effect of the temperature on our system, we remark in Eqs. (5) and (6) that the local and nonlocal dissipators depend on $T$ only through the factor $n$. As a consequence, since $n \rightarrow 0$ as $T \rightarrow 0 \mathrm{~K}$, lowering the environmental temperature tends to suppress the interactions between the atomic system and the EM thermal bath. On the other hand, the interaction strength $\Lambda_{j k}$ characterizing the dipole-dipole interaction between the atomic couple $(j, k)$ is temperature independent. Consequently, the hopping fluxes depend on $T$ only indirectly through the state $\rho$, and are much less affected by a change of the environmental temperature than the local and nonlocal ones. In particular, unlike the dissipative fluxes, the hopping ones do not vanish in the limit $T \rightarrow 0 \mathrm{~K}$.

This explains the difference between the two configurations of Fig. 2. Indeed, at $T=10 \mathrm{~K}$, the interactions between the atomic system and the EM bath are limited. As a consequence, the excitations pumped into $p$ are transmitted to atom 2 via hopping without almost any loss, and so forth until they reach the extraction site $e$. The interaction strength of the hopping fluxes $\Lambda_{i j}$ being a decreasing function of the distance between atoms $i$ and $j$, the case of the regular chain $(d=a)$ produces the best transport with $\chi \simeq 100 \%$. However, as $d$ increases, the interaction $\Lambda_{3 e}$ between atoms 3 and $e$ decreases, inducing a diminution of the hopping flux between them: the hopping-transmission chain weakens, resulting in an efficiency collapsing to 0 .

In the case $T=300 \mathrm{~K}$, the initial scenario $(d \simeq a=$ $0.1 \mu \mathrm{m}$ ) is quite similar, with $\chi \simeq 100 \%$ for a regular chain. However, contrary to the low-temperature scenario, pulling away the extraction atom results in a remarkable efficiency amplification. In particular, as pointed out previously, the efficiency reaches a maximum value greater than $900 \%$. This means that the increase of extracted energy from $e$ due to pumping is much larger than the energy injected into $p$. This suggests that the atomic system draws the additional excitations from the environmental thermal bath. A detailed investigation of this phenomenon will be the object of Sec. IV.

This dramatic efficiency amplification, of almost one order of magnitude, is the main result of this paper. We have shown that despite the simple 1D geometry of the system, a clever manipulation of thermal and geometrical parameters can be exploited to actively tune the amount of energy transported within the chain. Compared to previous works concerning 2D and 3D geometries [21], our 1D chain is able to produce an efficiency at least three times greater.

Moreover, the efficiency amplification we highlight is achievable in a wide range of geometrical configurations. More specifically, while for $T=10 \mathrm{~K}$ the only chain realizing $\chi \simeq 100 \%$ is the regular one, for $T=300 \mathrm{~K}$ an efficiency larger than $100 \%$ is produced within the entire interval $d \in[0.5,2] \mu \mathrm{m}$, including distances between the last two atoms up to 20 times greater than the regular-chain spacing. This proves that this long-range amplified energy transport 
is robust with respect to small displacements of atom $e$, and results indeed from a combination of thermal and geometrical features.

\section{DISCUSSION}

In this section, we unveil the physical mechanisms producing the efficiency amplification and the long-range transport. To this end, we remain focused on the case studied in the previous section, namely a four-atom chain. The efficiency amplification being a stationary effect, we begin this section with a steady-state analysis. After that, we look into the dynamics of the system to understand the establishment of the mechanisms entering into play.

In both Secs. IV A and IV B, the simulations have been realized with the temperature of the EM bath fixed at $T=$ $361 \mathrm{~K}$, which produces a maximum efficiency of $\chi \simeq 1000 \%$, with the rest of the parameters being the same as in Sec. III.

\section{A. Steady-state analysis}

According to Eq. (19), the efficiency characterizes the difference in the energy extracted from the chain between the scenarios with and without pumping. Therefore, in order to understand the origin of the efficiency amplification observed in the previous section, it is natural to investigate the effect of the pumping on the energy exchanges occurring both inside the atomic system and between the atoms and the bath. We start this section by introducing the quantities we use to study these effects. These are valid at any time $t$, but we will start our investigation by focusing on the steady state of the atomic system, i.e., the state reached for $t \rightarrow+\infty$. The analysis of the dynamics will be the object of Sec. IV B.

In order to characterize the effect of the pumping on the heat fluxes, we define the differences

$$
\begin{aligned}
\Delta \dot{Q}_{\mathrm{loc}}^{(j)} & =\dot{Q}_{\mathrm{loc}}^{(j)}-\dot{Q}_{\mathrm{loc}, 0}^{(j)}, \\
\Delta \dot{Q}_{\varphi}^{(j k)} & =\dot{Q}_{\varphi}^{(j k)}-\dot{Q}_{\varphi, 0}^{(j k)},
\end{aligned}
$$

where the index $\varphi \in\{$ nl,hop $\}$ according to the type of flux under investigation. The label 0 corresponds to the scenario in which no pumping is performed. We also define, for a given atom $j$,

$$
\Delta \dot{Q}_{\varphi}^{(j)}=\sum_{k \neq j} \Delta \dot{Q}_{\varphi}^{(j k)},
$$

which describes the total contribution of the type of flux under consideration involving atom $j$ (once again, $\varphi \in\{$ nl,hop $\}$ ).

In our linear chains, the number of heat fluxes grows very quickly with the number of atoms, and understanding the interplay between all of them can be tricky. For this reason, in the following, we track step by step the root of the mechanism producing efficiency amplification starting from general observations, so that the reader can follow the entire reasoning.

We begin our analysis by investigating the effect of pumping on each kind of flux involving atom $e$. Figure 3 shows $\Delta \dot{Q}_{\mathrm{loc}}^{(e)}$, $\Delta \dot{Q}_{\mathrm{nl}}^{(e)}$, and $\Delta \dot{Q}_{\mathrm{hop}}^{(e)}$ as a function of $d$ at stationarity normalized by $P$, the flux of energy pumped into the system (the extraction site is not show). As one can see, the hopping contribution has a behavior extremely similar to the one of the efficiency

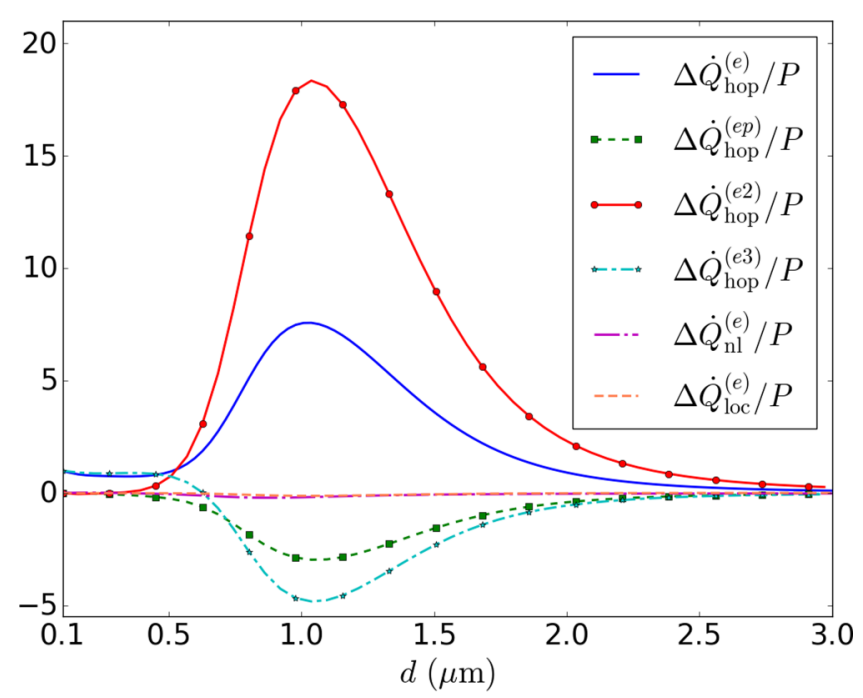

FIG. 3. Difference of the heat fluxes involving atom $e$ between the scenarios with and without pumping at the steady state divided by $P$. Each type of heat flux is represented: $\Delta Q_{\text {hop }}^{(e)}$ (solid blue line), $\Delta Q_{\mathrm{nl}}^{(e)}$ (long-dashed purple line), and $\Delta Q_{\mathrm{loc}}^{(e)}$ (dashed orange line). The contribution of each atom to $\Delta Q_{\mathrm{hop}}^{(e)}$ is also plotted: $\Delta Q_{\mathrm{hop}}^{(e p)}$ (dashed green line with squares), $\Delta Q_{\text {hop }}^{(e 2)}$ (solid red line with circles), and $\Delta Q_{\text {hop }}^{(e 3)}$ (dot-dashed cyan line with stars).

(see Fig. 2), while the local and nonlocal contributions are small with respect to the hopping one. In particular, having $\Delta \dot{Q}_{\mathrm{hop}}^{(e)}>0$ hints that the efficiency amplification arises from the enhancement (due to pumping) of the energy received by $e$ through hopping fluxes from the rest of the chain.

More specifically, unveiling the contribution of each atom to $\Delta \dot{Q}_{\text {hop }}^{(e)}$ provides further insight. Indeed, the hopping flux between atoms 2 and $e$ is clearly the most affected by the displacement of the latter. In particular, in the interval $d \in[0.5,2] \mu \mathrm{m}$, which is when efficiency amplification is realized, we have $\Delta \dot{Q}_{\text {hop }}^{(e 2)}>0$, meaning that the energy flowing from atom 2 to $e$ is increased in the presence of pumping. The opposite behavior occurs for the couples $(e, p)$ and $(e, 3)$. However, the variation is less pronounced than the one of $\Delta \dot{Q}_{\mathrm{hop}}^{(e 2)}$, which explains why the resulting hopping contribution verifies $\Delta \dot{Q}_{\text {hop }}^{(e)}>0$. Therefore, the efficiency amplification stems from the particularly enhanced hopping flux between atoms 2 and $e$. Note that in the regular-chain configuration $(d=0.1 \mu \mathrm{m})$, atom $e$ receives the additional energy due to pumping only by hopping from its closest neighbor, that is, atom 3 .

This leads us to investigate the reason why $\Delta \dot{Q}_{\mathrm{hop}}^{(e 2)}>0$. To this end, we plot in Fig. 4 the quantities defined in Eqs. (20) and (21) involving atom 2 at stationarity normalized by $P$.

In the interval of $d$ in which efficiency amplification is realized, the local flux of atom 2 is clearly enhanced, meaning that the presence of pumping increases the energy drawn locally by atom 2 from the bath, reaching a maximum $\simeq 15 P$ close to $d \simeq 1 \mu \mathrm{m}$. The nonlocal contributions $\Delta \dot{Q}_{\mathrm{nl}}^{(2)}$ are also increased, but are constant and relatively small compared to $\Delta \dot{Q}_{\mathrm{loc}}^{(2)}$ in the efficiency-amplification region. 


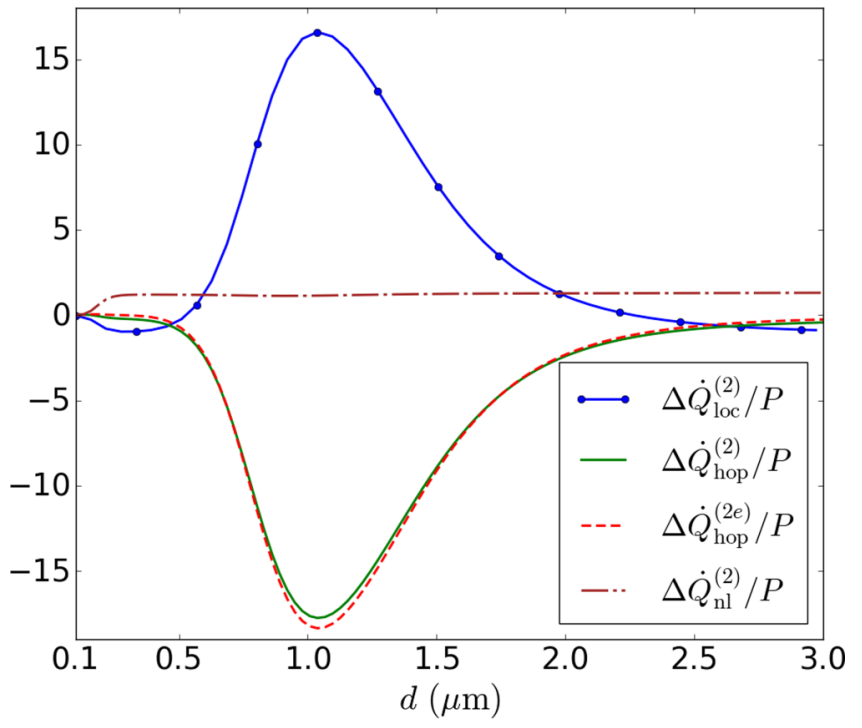

FIG. 4. Difference of the heat fluxes involving atom 2 between the pumping and no-pumping cases normalized by $P$ at the steady state. Each type of flux is plotted: $\Delta \dot{Q}_{\text {loc }}^{(2)}$ (solid blue line with circles), $\Delta \dot{Q}_{\text {hop }}^{(2)}$ (solid green line), and $\Delta \dot{Q}_{\mathrm{nl}}^{(2)}$ (dash-dotted brown line). The contribution of $\Delta \dot{Q}_{\text {hop }}^{(2 e)}$ (dashed red line) is also shown.

On the contrary, the hopping fluxes show a behavior opposed to the one of $\Delta \dot{Q}_{\text {loc }}^{(2)}$, highlighting the fact that the energy absorbed by atom 2 from the environment is almost entirely transmitted by hopping to the rest of the chain. Moreover, as illustrated by $\Delta \dot{Q}_{\mathrm{hop}}^{(2 e)}$, most of this energy is yielded to the extraction site $e$.

The previous observations illustrate the efficiencyamplification mechanism: in the presence of pumping, as the extraction site is moved away from the rest of the chain, the energy absorbed by atom 2 from the EM bath is significantly increased with respect to the no-pumping scenario. Most of this energy is transmitted to atom $e$ through hopping flux and results in an efficiency $\chi \geqslant 100 \%$, meaning that the additional energy extracted from the chain is greater than the one injected in site $p$.

The behavior of the local flux of atom 2 being modified in the presence of pumping suggests that the other atoms might be subject to a change in their interaction with the EM bath as well. To explore this possibility, we plot in Fig. 5 the difference of the local and nonlocal heat fluxes of atoms $p$ and 3 between the scenarios with and without pumping, as a function of the displacement of $e$.

As $d$ increases, both atoms experience a similar diminution of their local fluxes which reaches a constant value when $e$ is far enough. On the other hand, $\Delta \dot{Q}_{\mathrm{nl}}^{(p 3)}$ has an analogous behavior but with an opposite sign. As a consequence, the energy loss occurring through the local channels $\dot{Q}_{\text {loc }}^{(p)}$ and $\dot{Q}_{\text {loc }}^{(3)}$ is almost entirely counterbalanced by the absorption of excitations through their nonlocal flux.

So far, the analysis of the fluxes gives us an interpretation of the efficiency-amplification mechanism. Pumping excitations into the chain results in a reorganization of the interactions between the atoms and the environmental EM bath, to the benefit of the efficiency.

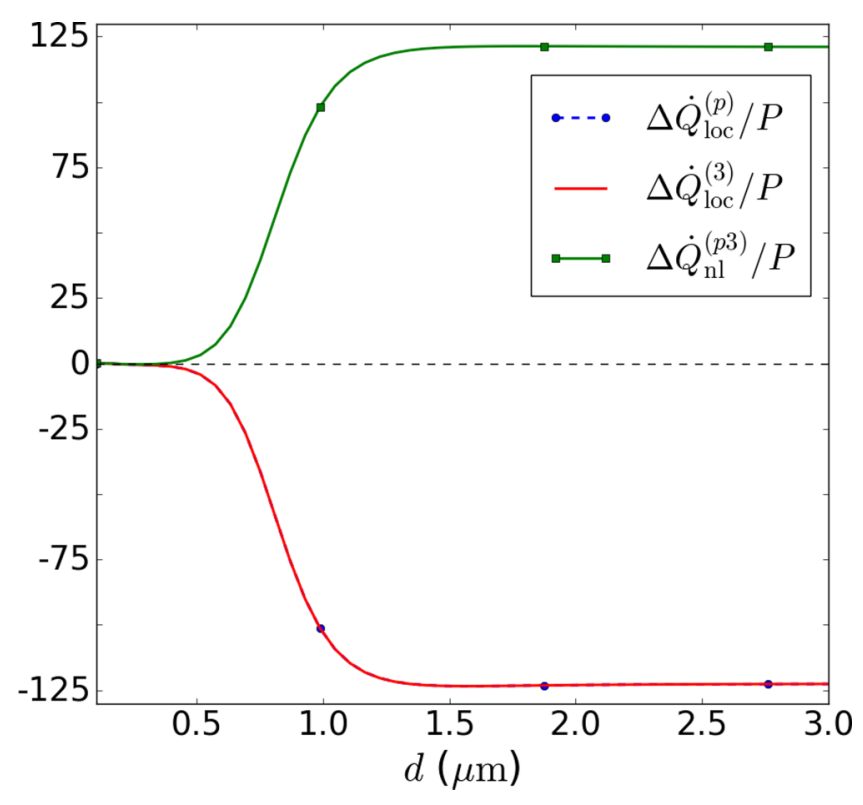

FIG. 5. Difference between the pumping and no-pumping cases of the local heat fluxes of the atoms $p\left(\Delta \dot{Q}_{\text {loc }}^{(p)}\right.$, dashed blue line with circles) and $3\left(\Delta \dot{Q}_{\text {loc }}^{(3)}\right.$, solid red line), and of the nonlocal flux between them $\left(\Delta \dot{Q}_{\mathrm{nl}}^{(p 3)}\right.$, solid green line with squares), as a function of the displacement of $e$. These quantities are normalized by the energy flux pumped into the system.

More specifically, the triplet $(p, 2,3)$ plays the role of an excitation injector: the collective interaction of the couple $(p, 3)$ has the effect of singling out atom 2 , which sees its locally drawn energy improved. The energy absorbed by the triplet is then transmitted to the extraction site $e$ via hopping, with, notably, a particularly enhanced flux of the pair $(2, e)$. As a result, the energy extracted from $e$ is not only greater than in the no-pumping scenario, but of an amount which can go largely beyond the energy injected in the chain.

According to this interpretation, the reasons why amplified efficiency occurs over long ranges, along with the nonmonotonic behavior of the efficiency as a function of $d$, both become clear. On the one hand, the excitation injector needs to be relatively isolated from the rest of the system; otherwise the symmetry of this triplet is broken, resulting in a different heat-flux distribution and thus in the disappearance of the effect. On the other hand, if the triplet $(p, 2,3)$ is too far away from the rest of the chain, the hopping fluxes will be diminished, resulting in a lowered efficiency. Therefore, the optimal displacement of $e$ stems from the best compromise between the confinement of the excitation injector and its proximity to the extraction site.

Thus, in this section, the investigation of the effect of pumping on the heat-flux distribution has led us to identify the first three atoms of the chain as an excitation injector. As the name suggests, the effect of this triplet is to inject excitations within the atomic chain, resulting in an enhancement of the energy extracted from the chain. The reason why this efficiency amplification occurs over long range stems from the geometrical condition required for the establishment of the mechanism of the excitation injector. 


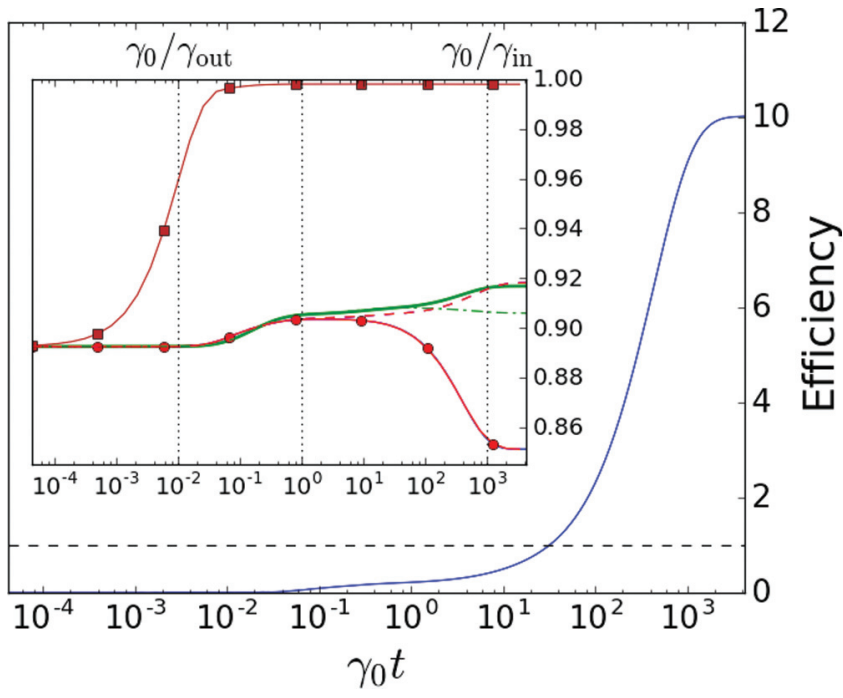

FIG. 6. Time evolution of the efficiency $\chi$ (solid blue line). Inset: Ground-state population of each atom (index 0 corresponds to the no-pumping scenario): $p^{(p)}$ (solid red line with circles), $p_{0}^{(p)}$ (dashed red line), $p^{(2)}$ (solid green line), $p_{0}^{(2)}$ (dot-dashed green line), and $p^{(e)}$ (solid brown line with squares). The curves $p^{(3)}, p_{0}^{(3)}$, and $p_{0}^{(e)}$ coincide with $p^{(p)}, p_{0}^{(p)}$, and $p^{(e)}$, respectively.

\section{B. Dynamics}

Having unveiled the mechanism producing the long-range energy-transport efficiency amplification at the steady state, we dedicate this section to the dynamics of our system. The analysis of the different time scales of the system will allow us to understand the apparition of the different channels of energy exchanges, and, in particular, to identify the establishment over time of the excitation injector. Throughout this part, the distance between the extraction site and atom 3 is fixed at $d=1.03 \mu \mathrm{m}$, resulting in an efficiency of $\chi=1021 \%$.

First, to have an overview of the dynamics of the system, let us begin this analysis with Fig. 6 showing the time evolution of the efficiency as well as the ground-state population of each atom of the chain. It becomes clear from the plots that the latter evolves according to three different time scales. The first one is due to the extraction, characterized by the rate $\gamma_{\text {out }}$, which occurs very early and drives the population of the atom $e$ close to $p^{(e)} \simeq 1$ similarly in both cases, i.e., with and without pumping $\left(p^{(e)}=p_{0}^{(e)}\right.$, with the index 0 referring to quantities in the absence of pumping).

The second time scale entering into play triggers the evolution of the population of atoms $p, 2$, and 3 and is described by the local $\left(\gamma_{j}\right)$ and nonlocal $\left(\gamma_{j k}\right)$ rates. These are of the order of magnitude of $\gamma_{0}$, which is the only one represented in the inset of Fig. 6. It is worth stressing that the ground-state population of atoms $p$ and 3 has the same evolution whether the pumping is performed $\left(p^{(p)}=p^{(3)}\right)$ or not $\left(p_{0}^{(p)}=p_{0}^{(3)}\right)$, and that in both scenarios, this evolution is different from the one of the ground-state population of atom 2 .

In addition, the populations of each atom have a different dynamics between the two scenarios $p^{(j)} \neq p_{0}^{(j)}$ (except for the atom $e$, as mentioned before). Not surprisingly, this difference

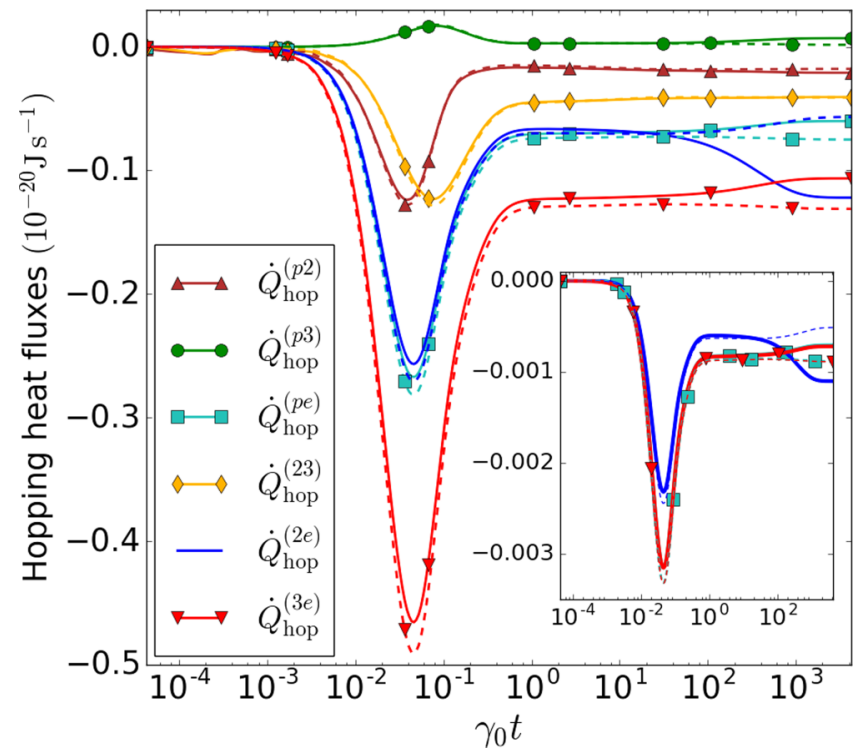

FIG. 7. Time evolution of the hopping fluxes for each couple of atoms in the presence of pumping. Inset: Imaginary part of the coherences of couples $(p, e),(2, e)$, and $(3, e)$ also in the presence of pumping, with the same color scheme. The curves of pairs $(p, e)$ and $(2, e)$ are superimposed. The dotted lines, both in the main part of the figure and in the inset, represent the same quantities in the absence of pumping.

becomes significant only at the third time scale of the system, determined by the pumping rate $\gamma_{\text {in. }}$. In particular, the stationary ground-state populations of atoms $p$ and 3 in the no-pumping scenario are greater than the one of atom $2\left(p_{0}^{(p)}=p_{0}^{(3)}>\right.$ $\left.p_{0}^{(2)}\right)$, while the situation is reversed in the pumping case $\left(p^{(2)}>p^{(p)}=p^{(3)}\right)$. In other words, the presence of pumping has a notable effect on the population distribution of these atoms, which suggests why the efficiency is increasing the most at this stage of the evolution.

In order to explain this modification in the distribution of the ground-state populations, we now turn our investigation toward the dynamics of the heat fluxes. We start with Fig. 7, depicting the time evolution of the hopping ones. According to Eq. (13), the dynamics of these energy exchanges is driven by the one of the imaginary part of the coherences, as confirmed by the inset.

The peaks appearing in the interval $\left[10^{-2}, 10^{-1}\right]$ stem from the fact that the atom $e$ is in its ground state $\left(p^{(e)} \simeq 1\right)$ due to extraction. As a consequence, coherences raise between subparts of the atomic system in order to draw it closer to its Gibbs state, thus producing hopping fluxes within the chain. Note that the ones with the greatest amplitudes are the fluxes involving atom $e$. The decrease of these peaks coincides with the appearance of the local and nonlocal heat fluxes whose dynamics is shown in Figs. 8 and 9.

Concerning the local heat fluxes, note that $\dot{Q}_{\text {loc }}^{(e)}$ begins its evolution earlier than the rest of the others because of the extraction. Moreover, $\dot{Q}_{\mathrm{loc}}^{(e)}>0$ implies that this flux competes with the loss of energy induced by $\gamma_{\text {out }}$. Later in the evolution, the local and nonlocal fluxes of the other atoms enter into play. Note that only the couples $(p, 3)$ and $(2,3)$ produce nonlocal 


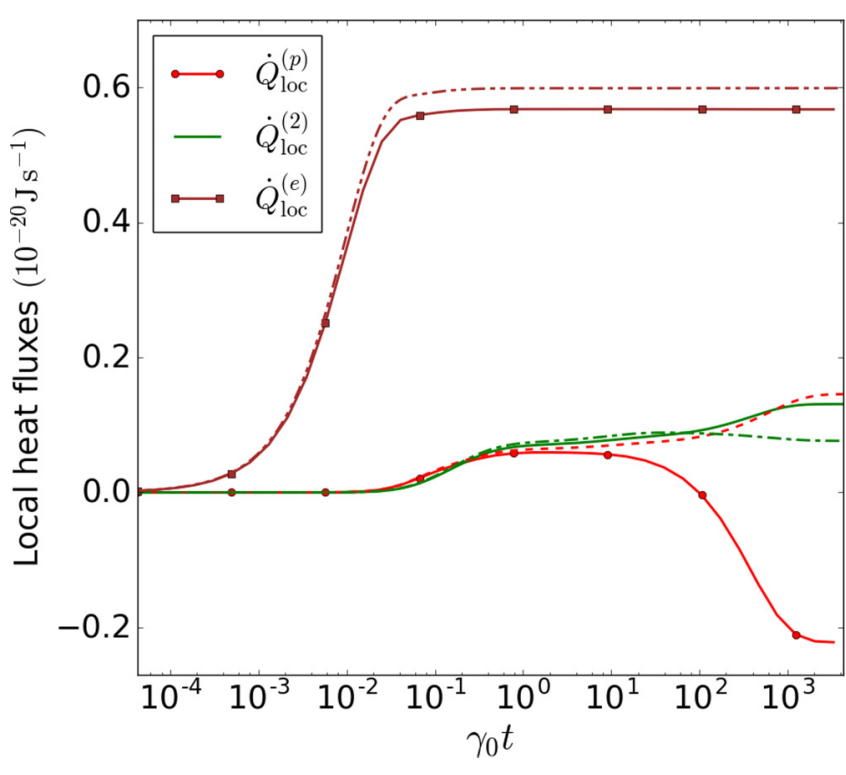

FIG. 8. Time evolution of the local heat fluxes of each atom of the chain in the presence of pumping: $\dot{Q}_{\mathrm{loc}}^{(p)}$ (solid red line with circles), $\dot{Q}_{\mathrm{loc}}^{(2)}$ (solid green line), and $\dot{Q}_{\mathrm{loc}}^{(e)}$ (solid brown line with squares). The dotted lines are the same quantities in the absence of pumping: $\dot{Q}_{\mathrm{loc}, 0}^{(p)}$ (dashed red line), $\dot{Q}_{\mathrm{loc}, 0}^{(2)}$ (dot-dashed green line), and $\dot{Q}_{\text {loc, } 0}^{(e)}$ (double-dot-dashed brown line). The curves describing $\dot{Q}_{\mathrm{loc}}^{(3)}$ and $\dot{Q}_{\mathrm{loc}, 0}^{(3)}$ coincide with those of $\dot{Q}_{\mathrm{loc}}^{(p)}$ and $\dot{Q}_{\mathrm{loc}, 0}^{(p)}$, respectively.

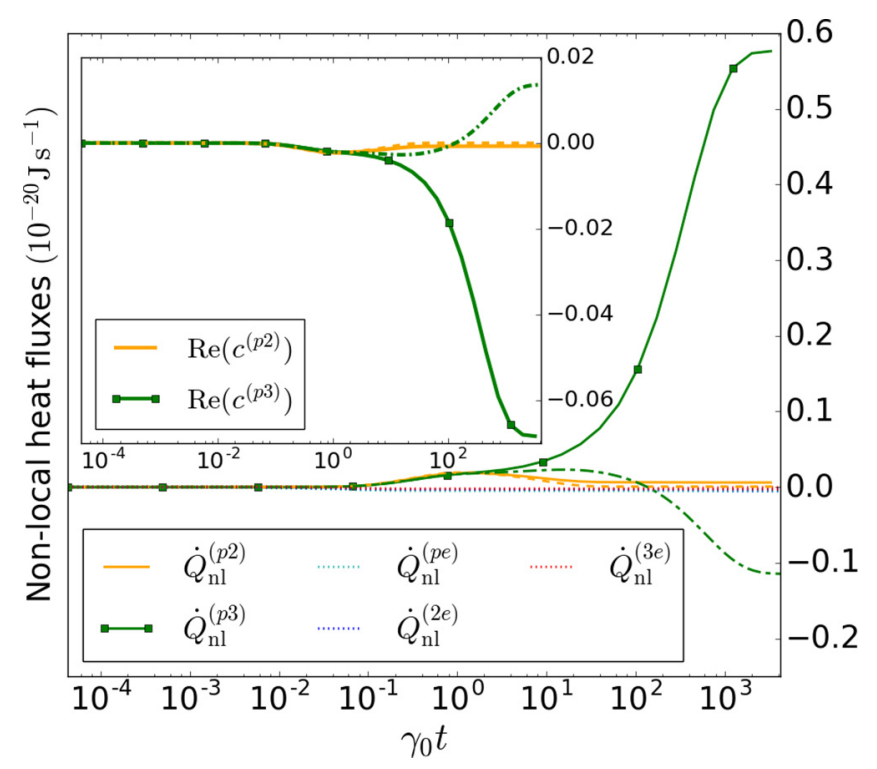

FIG. 9. Dynamics of the nonlocal fluxes for each pair of atoms composing the chain. Inset: Real part of the coherences of the couples $(p, 2)$ (solid orange line with pumping, dashed orange line without pumping) and $(p, 3)$ (solid green line with squares with pumping, dot-dashed green line without pumping). Main part: Nonlocal fluxes between qubits. The same color scheme is used for the couples $(p, 2)$ and $(p, 3)$. Both in the inset and in the main part, the results for the couple $(2,3)$ coincide with the ones of $(p, 2)$. In the main part, all of the remaining heat fluxes (almost negligible and unchanged with and without pumping) are traced with dotted lines. contributions, and that they are driven by the real part of the coherences (inset of Fig. 9).

The main distinction between the pumping and no-pumping scenarios occurs at the third time scale. The local fluxes of atoms $p$ and 3 become negative, meaning that they are both losing energy. However, the nonlocal flux stemming from the collective interaction between them increases significantly in the pumping case, such that the resulting flux between the field and this pair of atoms is positive: $\dot{Q}_{\mathrm{nl}}^{(p 3)}+\dot{Q}_{\mathrm{loc}}^{(p)}+\dot{Q}_{\mathrm{loc}}^{(3)}>0$. In other words, the subsystem $(p, 3)$ is absorbing more energy than in the no-pumping scenario, which explains why their ground-state population is lowered.

In addition, the local flux of atom 2 increases with respect to the no-pumping case. Yet, its ground-state population is the closest one to $p^{(e)}$ and the imaginary parts of the coherences of the pair $(2, e)$ are also increased, inducing an enhanced hopping flux between these two atoms. On the contrary, the amplitudes of $\dot{Q}_{\mathrm{hop}}^{(p e)}$ and $\dot{Q}_{\mathrm{hop}}^{(3 e)}$ are lowered, but remain positive, meaning that atoms $p$ and 3 keep yielding energy to the extraction site (see Fig. 7). This analysis suggests a complicated three-body coupled dynamics, in which two symmetric atoms build up correlations leaving to the central atom more freedom to strongly interact with the thermal environment. The description in terms of physical quantities pertaining to subparts of such a three-body system, on the one hand provides a direct and clearer physical picture, but on the other hand hinders the full complexity of this phenomenon. However, a complete study of the complicated structure of the interplay between atom-atom coherences and collective atom-environment coupling is beyond the goal of this work.

\section{GENERAL STUDY OF EFFICIENCY AMPLIFICATION AND LONG-RANGE TRANSPORT}

\section{A. Variation of relevant parameters}

Thus far, we have studied the efficiency amplification and long-range transport in the case of a four-atom chain with specific parameters. We dedicate the first part of this section to the analysis of the robustness of these two effects when relevant parameters of the system (frequency, temperature, coordinate of the last atom) are changed, still with a four-atom chain. The second part, on the other hand, is devoted to the study of chains composed with a different number of atoms.

We start our investigation with Fig. 10, where we analyze the dependence of the efficiency amplification on different parameters. More specifically, Figs. 10(a)-10(d) are density plots of $\chi$ as a function of $d$ and of the temperature $T$ for different transition frequencies.

The first feature to point out is that Figs. 10(a)-10(c) show efficiency-amplified regions $(\chi>100 \%)$, witnessing a nonmonotonic behavior of $\chi$ with respect to both parameters $d$ and $T$, absent in Fig. 10(d). Note that in some regions, the efficiency takes negative values, meaning that pumping excitations into the system reduces the energy extracted from the chain $\left(E<E_{0}\right)$.

Moreover, we must stress that the values taken by the efficiency are very different for each panel of Fig. 10, showing a strong dependence of this effect on the atomic transition frequency. 

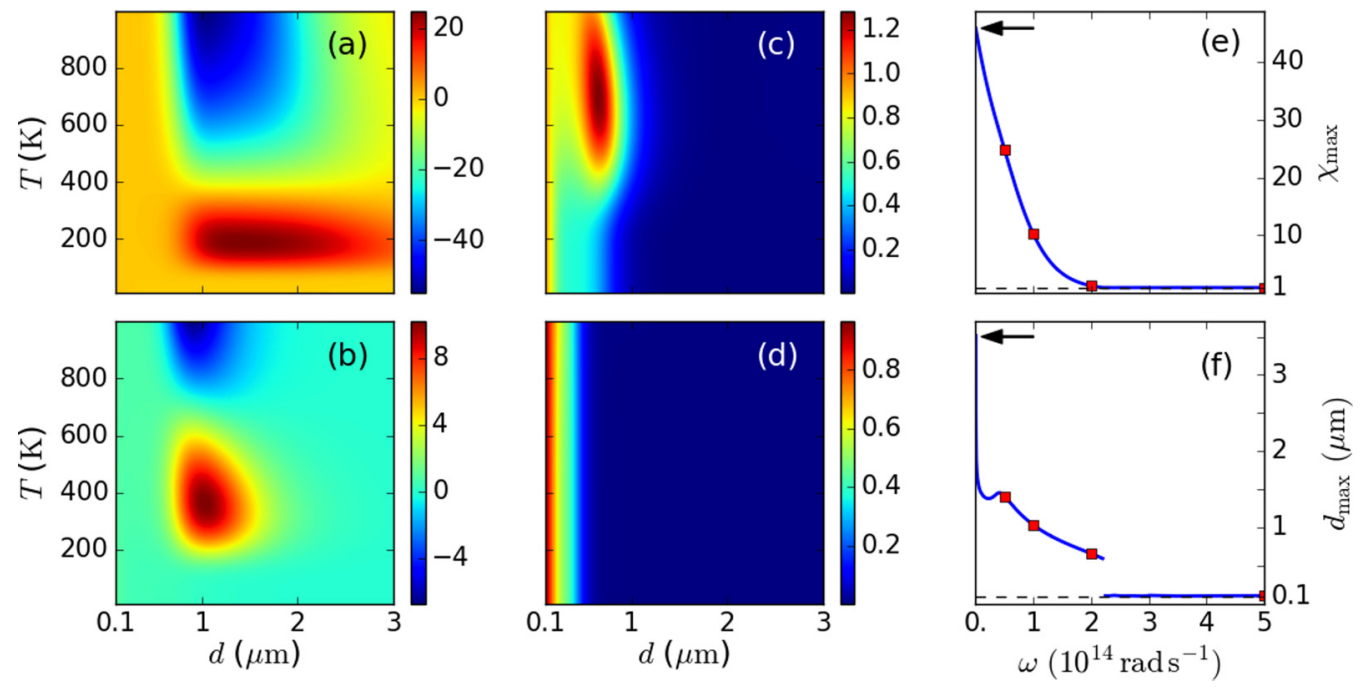

FIG. 10. (a)-(d) Efficiency as a function of the distance between atom $e$ and the rest of the chain and of the bath temperature for different frequencies: (a) $\omega=0.5 \times 10^{14} \mathrm{rad} \mathrm{s}^{-1}$, (b) $\omega=1 \times 10^{14} \mathrm{rad} \mathrm{s}^{-1}$, (c) $\omega=2 \times 10^{14} \mathrm{rad} \mathrm{s}^{-1}$, and (d) $\omega=5 \times 10^{14} \mathrm{rad} \mathrm{s}{ }^{-1}$. (e) Maximum efficiency and (f) distance at which this maximum is realized, as a function of the frequency. The maximization has been performed with respect to the parameters $d \in[0.1,3] \mu \mathrm{m}$ and $T \in[10,1000] \mathrm{K}$. The black arrows in (e) and (f) indicate the asymptotic limit of respectively $\chi_{\max }$ and $d_{\max }$ at low frequencies.

In order to have a deeper insight on this dependence, Fig. 10(e) shows the maximum efficiency with respect to both variables $d \in[0.1,3] \mu \mathrm{m}$ and $T \in[10,1000] \mathrm{K}$ as a function of $\omega$. Remarkably, at frequencies around $\omega \simeq 0.1 \times$ $10^{14} \mathrm{rad} \mathrm{s}^{-1}$, the efficiency reaches $\chi_{\max } \simeq 4000 \%$. Then it decreases when increasing $\omega$, until reaching values $\leqslant 100 \%$, meaning that the efficiency amplification is no longer realized at frequencies $\geqslant 2 \times 10^{14} \mathrm{rad} \mathrm{s}^{-1}$.

In the asymptotic limit of low frequencies, indicated by black arrows in Figs. 10(e) and 10(f), the efficiency reaches a plateau at $\chi_{\max } \simeq 4500 \%$ for $\omega \leqslant 0.01 \times 10^{14} \mathrm{rad} \mathrm{s}^{-1}$ [28].

To have a deeper insight into the pumping and extraction fluxes entering into play, Fig. 11 pictures the quantities from which $\chi_{\max }$ of Fig. 10 is obtained in the region where $\chi_{\max }>$ $100 \%$. As one can see, we have $P_{\chi_{\max }} \ll E_{\chi_{\max }}$, and increasing the frequency results in increasing both $P_{\chi_{\max }}$ and $E_{\chi_{\max }}$. These two features stem, respectively, from the facts that $\gamma_{\text {in }} \ll \gamma_{\text {out }}$ and that both of these coefficients depend on $\gamma_{0}$.

Concerning the position of the maximum of efficiency in Figs. 10(a)-10(d), one can notice that increasing the frequency increases the temperature $T_{\chi_{\max }}$ at which $\chi_{\max }$ is reached. In the configurations such that $\chi_{\max }>100 \%, T_{\chi_{\max }}(\omega)$ follows a linear behavior (not shown here) with respect to $\omega$. This behavior is close to that of the temperature $T_{B_{\max }}(\omega)$, which produces the maximum of the spectral radiance $B$ of the blackbody radiation given by Planck's law: $B(\omega, T)=n(\omega, T) h \omega^{3} / 4 \pi^{3} c^{2}$. In other words, we have $T_{\chi_{\max }}(\omega) \simeq T_{B_{\max }}(\omega)$, which shows that the efficiency amplification is indeed the consequence of thermal effects. This similarity is due to the fact that the thermal dependence of the state of our system stems from the presence of the local and nonlocal dissipators in the master equation, in which the temperature acts only through the factor $n$, similarly to $B$.

Moreover, when increasing the frequency, the distance of the last atom for which $\chi_{\max }$ is reached approaches that of the regular-chain configuration. To study this phenomenon in more detail, we plot in Fig. 10(f) the displacement of atom $e$ at which $\chi_{\max }$ is reached as a function of the frequency. Clearly, the distance $d_{\max }$ decreases when increasing $\omega$. The discontinuity at $\omega \simeq 2.3 \times 10^{14} \mathrm{rad} \mathrm{s}^{-1}$ witnesses the transition from the long-range energy-transport configuration, in which the efficiency is amplified, to the regular-chain one, where $\chi_{\max } \leqslant 100 \%$. In other words, while for $\omega \gtrsim 2.3 \times$ $10^{14} \mathrm{rad} \mathrm{s}^{-1}$ the best efficiency $(\leqslant 100 \%)$ is always realized by the regular configuration $(d=a)$, for any lower frequencies

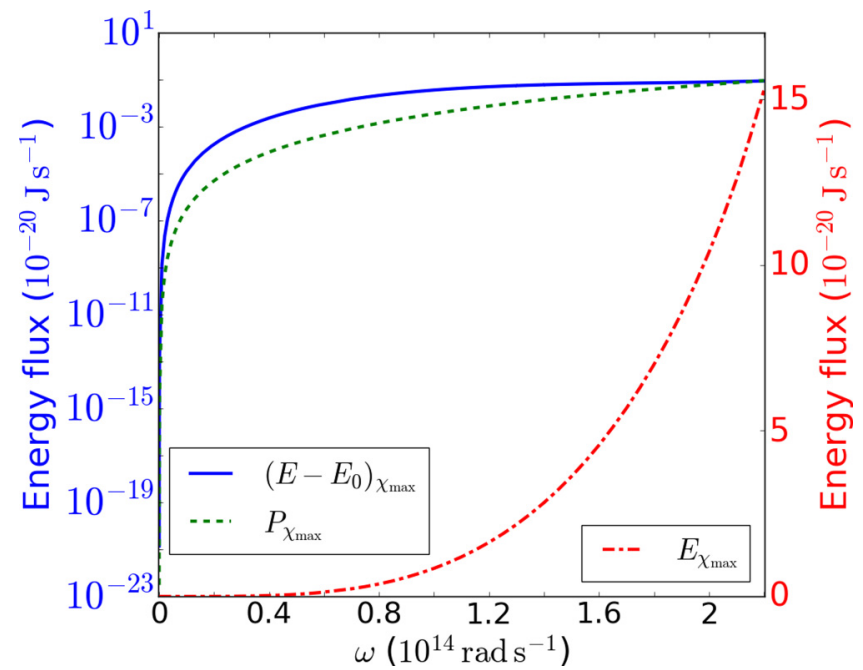

FIG. 11. Left vertical scale: difference $\left(E-E_{0}\right)_{\chi_{\max }}$ of the energy extracted from the chain between the scenarios with and without pumping (solid blue line) and $P_{\chi_{\max }}$ of the energy pumped into the chain (dashed green line). Right vertical scale: $E_{\chi_{\max }}$ (dot-dashed red line) of the energy extracted from the chain in the pumping case. All of the quantities are plotted as a function of the frequency and correspond to the maximum of efficiency for every frequency, i.e., $\left(E-E_{0}\right)_{\chi_{\max }} / P_{\chi_{\max }}$ results in $\chi_{\max }$ plotted in Fig. 10(e). 
we observe an optimized distance $d_{\text {max }}$ producing a long-range efficiency amplification.

\section{B. More atoms}

Having studied in detail the four-atom configuration, we have shown in Sec. IV that the excitation injector, i.e., the triplet of the first three atoms of the chain, is a key ingredient to observe long-range efficiency amplification.

First, we begin our analysis by reproducing Fig. 2 with $N=$ $2, \ldots, 7$. This means that for each $N$, we have a linear chain $\{p, \ldots, N-1, e\}$ (reducing to $\{p, e\}$ in the case of two atoms). The distance between two neighbors is $a=0.1 \mu \mathrm{m}$, except the one between atoms $(N-1, e)$ that we call $d$. Following Fig. 2, we plot the efficiency as $d$ increases, i.e., when the extraction site is moved away from the rest of the chain, for the two temperatures $T=10$ and $T=300 \mathrm{~K}$. All of the parameters being the same as in Fig. 2, the case $N=4$ is strictly the same in both figures.

For $T=10 \mathrm{~K}$, the behavior we observe for any $N$ is qualitatively the same as the one we already had for $N=4$ : an efficiency starting from $100 \%$ and collapsing to 0 as $d$ increases.

On the contrary, for $T=300 \mathrm{~K}$, we clearly see that $N=4$ is the only configuration showing a strong amplification of the efficiency. As we explained before, the excitation injector needs to be relatively isolated from the rest of the chain to be effective; otherwise its mechanism is perturbed by energy exchanges with other atoms. In Fig. 12, the configuration $N=$ 4 is the only one to provide this condition for appropriate values of $d$, which explains why it is the only one showing an amplified efficiency.
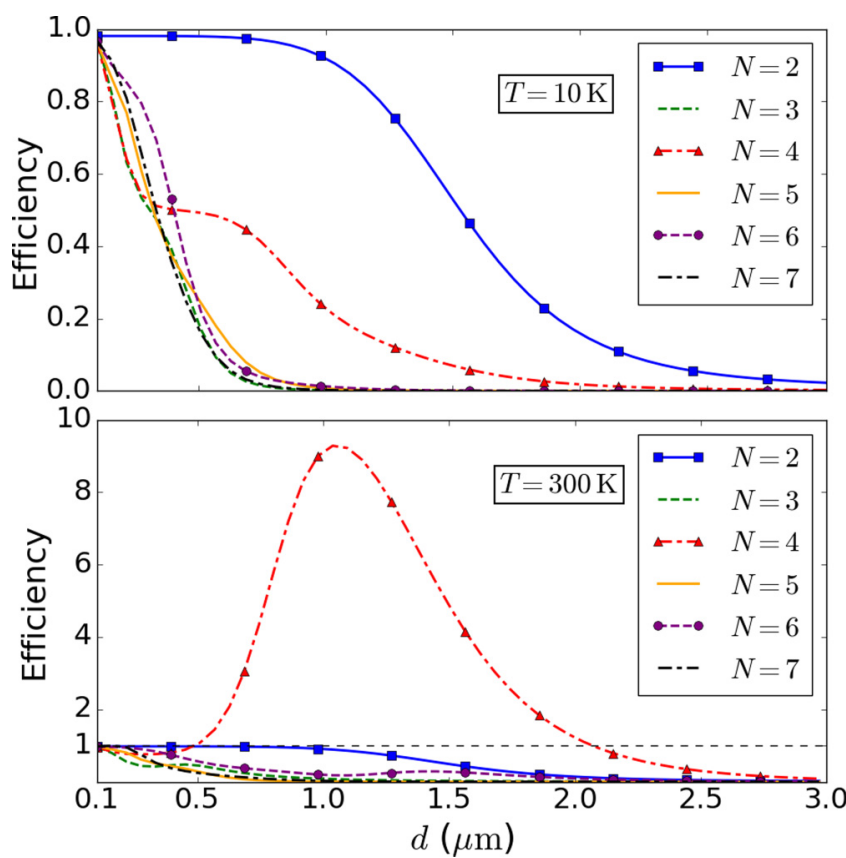

FIG. 12. Efficiency as a function of the distance between atom $e$ and atom $N-1$ for different values of $N$ (total number of atoms composing the chain; see legend). The upper plot is realized at $T=$ $10 \mathrm{~K}$ and the lower one at $T=300 \mathrm{~K}$.

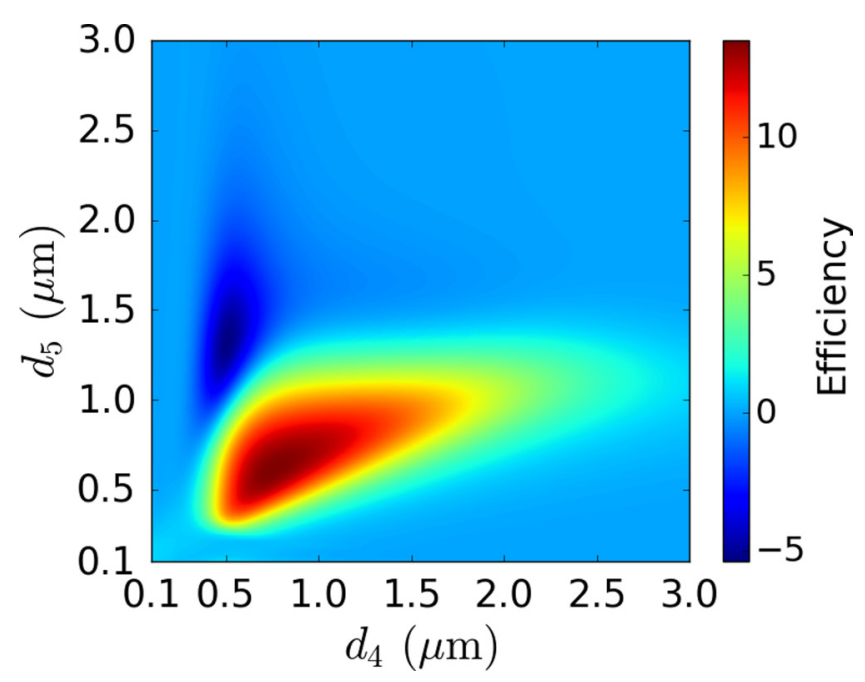

FIG. 13. Density plot of the efficiency as a function of the displacement of atoms 4 and $e$ in a five-atom linear chain. The other atoms are regularly separated by a distance $a=0.1 \mu \mathrm{m}$.

More specifically, in the case $N=3$, the symmetry of the excitation injector $(p, 2, e)$ is broken since atom $e$ is moved away. On the other hand, for $N>4$, the role of the excitation injector being played by $(p, 2,3)$ is broken due to the presence of atom 4 , which is too close to atom 3 .

With this information in mind, we have explored geometrical configurations with $N>4$ atoms in which the effect of the excitation injector is not killed. To this end, we allowed displacements not only of atom $e$, but also of any atom which does not belong to the triplet $(p, 2,3)$. In the following simulations, the atomic frequency is $\omega=1 \times 10^{14} \mathrm{rad} \mathrm{s}^{-1}$ and the temperature is fixed at $T=361 \mathrm{~K}$.

For the configuration $N=5$, Fig. 13 is a density plot of the efficiency as a function of the displacement of both atoms 4 and $e$ in the chain $\{p, 2,3,4, e\}$. There is clearly a region where long-range efficiency amplification is realized.

TABLE I. Maxima of the efficiency for linear chains with different number of atoms. These maxima have been obtained with a genetic algorithm (GA) and are either global $(N=4,5)$ or local $(N=6,7)$. In each simulation, the variables are the displacement $d_{j}$ along the $x$ axis of the $j$ th atom with respect to its regular position, and such that $j \notin(p, 2,3)$. These three atoms are regularly distributed with a separation of $a=0.1 \mu \mathrm{m}$. Concerning the parameters used for the GA, the initial population of each iteration was $\mathrm{p}_{\mathrm{GA}}=10^{3}$, with only the best half of it surviving. The mutation rate was fixed at $r_{\mathrm{GA}}=20 \%$, and convergence has been reached when the relative difference between each couple of the first 20 positions as well as the difference between their efficiencies were, at most, $10^{-3}$, except for $N=7$ for which it was $10^{-2}$.

\begin{tabular}{cccccc}
\hline \hline$N$ & $d_{4}(\mu \mathrm{m})$ & $d_{5}(\mu \mathrm{m})$ & $d_{6}(\mu \mathrm{m})$ & $d_{7}(\mu \mathrm{m})$ & $\chi$ \\
\hline 5 & 0.763 & 0.618 & & & 13.518 \\
6 & 0.556 & 1.076 & 0.578 & & 13.982 \\
& 0.782 & 0.564 & 0.518 & & 13.631 \\
7 & 0.768 & 0.581 & 0.380 & 0.419 & 13.908 \\
\hline \hline
\end{tabular}


Moreover, Table I shows maxima of $\chi$ after displacement of the atoms that do not belong to the excitation injector in the case of $N$-atom chains $(N \in\{5,6,7\})$. They have been computed with the use of a genetic algorithm [29]. In the cases $N=4,5$, these maxima are global, while they are local for the configurations with $N=6,7$ atoms. As one can see, for $N>4$, the long-range efficiency amplification is even higher than the four-atom configuration. In particular, in the case of the seven-atom chain, the efficiency can reach values up to $1390 \%$, which is $\sim 1.4$ times greater than the maximum of the four-atom chain $(1000 \%)$. In addition, the transport is performed over longer distances.

Finally, the presence of multiple local maxima of the efficiency for $N=6,7$ indicates that having more and more atoms in the chain can provide geometrical flexibility to produce highly efficient energy transport over large distances. Indeed, with an increase in the number of channels of energy exchanges, the number of geometrical configurations for which the interplay of the fluxes produces a local maximum of efficiency is increased as well.

\section{CONCLUSIONS}

We studied energy transport within a chain of two-level quantum emitters weakly coupled to an environmental blackbody radiation. In particular, we investigated the efficiency of energy transport when excitations are incoherently pumped from one edge of the chain and extracted at the other one. As the main result, we have highlighted the remarkable appearance of a strongly amplified efficiency occurring for long-range transport.

More specifically, we have shown that this phenomenon is produced for nonregular configurations at finite temperature and results in an efficiency greater than the one observed with a regular chain at low $T$. In particular, efficiency can reach values far greater than $100 \%$ (e.g., $\chi \simeq 1400 \%$ ), meaning that the chain harvests additional energy from the bath during the transport process. Moreover, the length of the chain producing this amplified efficiency is remarkably greater than the regular configuration, thus allowing energy transport over longer distances.

We have also investigated, in the particular case of a four-atom chain, the robustness of efficiency amplification with respect to relevant parameters of the system, such as the temperature, the atomic frequency, and the distance of the extraction site. We have shown that this effect can be produced for a relatively wide range of temperatures and distances.

The investigation of the heat fluxes both at the steady state and during the time evolution of the atomic system has provided an interpretation for the mechanism producing this amplification: the elementary block composed of the first three atoms of the chain plays the role of an excitation injector by absorbing energy from the environment. More specifically, the atoms $(p, 3)$ collectively draw excitations through nonlocal flux, while in addition atom 2 absorbs energy locally. The energy absorbed by the triplet is transmitted to the extraction site mainly through hopping fluxes.

We have also explored systems composed of $N=5,6,7$ atoms, where we provided specific examples of configurations producing a long-range efficiency amplification. Two encouraging results stem from this study when compared to the more elementary four-atom chain: the efficiency can reach higher values ( $\sim 1.4$ times greater) and the chains producing such high efficiency also transport energy over distances larger than the regular configuration.

Further investigations with an even larger number of atoms would be insightful, notably in the case of linear chains composed of multiple excitation injectors. Besides, the longrange efficiency amplification discussed in this paper could possibly be experimentally observed with, e.g., Rydberg atoms [30] or quantum dots [31-35] playing the role of the quantum emitters.

\section{ACKNOWLEDGMENT}

The authors acknowledge financial support from the Julian Schwinger Foundation.
[1] Y. Dubi and M. Di Ventra, Rev. Mod. Phys. 83, 131 (2011).

[2] A. Ishizaki and G. R. Fleming, Annu. Rev. Condens. Matter Phys. 3, 333 (2012).

[3] N. Li, J. Ren, L. Wang, G. Zhang, P. Hänggi, and B. Li, Rev. Mod. Phys. 84, 1045 (2012).

[4] B. Leggio, B. Bellomo, and M. Antezza, Phys. Rev. A 91, 012117 (2015).

[5] P. Doyeux, B. Leggio, R. Messina, and M. Antezza, Phys. Rev. E 93, 022134 (2016).

[6] S. M. Menke and R. J. Holmes, Energy Environ. Sci. 7, 499 (2014).

[7] L. Wang and B. Li, Phys. Rev. Lett. 99, 177208 (2007).

[8] T. Brixner, J. Stenger, H. M. Vaswani, M. Cho, R. E. Blankenship, and G. R. Fleming, Nature (London) 434, 625 (2005).

[9] S. Lloyd and M. Mohseni, New J. Phys. 12, 075020 (2010).

[10] D. Manzano, PloS One 8, e57041 (2013).
[11] G. L. Celardo, G. G. Giusteri, and F. Borgonovi, Phys. Rev. B 90, 075113 (2014).

[12] J. Feist and F. J. Garcia-Vidal, Phys. Rev. Lett. 114, 196402 (2015).

[13] M. Mohseni, P. Rebentrost, S. Lloyd, and A. Aspuru-Guzik, J. Chem. Phys. 129, 174106 (2008).

[14] A. W. Chin, A. Datta, F. Caruso, S. F. Huelga, and M. B. Plenio, New J. Phys. 12, 065002 (2010).

[15] A. G. Dijkstra and Y. Tanimura, New J. Phys. 14, 073027 (2012).

[16] C. Wang, J. Ren, and J. Cao, Sci. Rep. 5, 11787 (2015).

[17] J. Schachenmayer, C. Genes, E. Tignone, and G. Pupillo, Phys. Rev. Lett. 114, 196403 (2015).

[18] A. N. Poddubny, Phys. Rev. B 92, 155418 (2015).

[19] C. Gonzalez-Ballestero, J. Feist, E. Moreno, and F. J. GarciaVidal, Phys. Rev. B 92, 121402 (2015).

[20] A. Bermudez, M. Bruderer, and M. B. Plenio, Phys. Rev. Lett. 111, 040601 (2013). 
[21] B. Leggio, R. Messina, and M. Antezza, Europhys. Lett. 110, 40002 (2015).

[22] H.-P. Breuer and F. Petruccione, The Theory Of Open Quantum Systems (Oxford University, Berlin, 2002).

[23] B. Bellomo and M. Antezza, New J. Phys. 15, 113052 (2013).

[24] R. Balian, From Microphysics To Macrophysics: Methods and Applications of Statistical Physics (Springer-Verlag, Berlin, 1991).

[25] J. Gemmer, M. Michel, and G. Mahler, Quantum Thermodynamics: Emergence of Thermodynamic Behavior Within Composite Quantum Systems (Springer, Berlin, 2010).

[26] J. Johansson, P. Nation, and F. Nori, Comput. Phys. Commun. 183, 1760 (2012).

[27] J. Johansson, P. Nation, and F. Nori, Comput. Phys. Commun. 184, 1234 (2013).

[28] From a technical point of view, the master equation becomes particularly difficult to solve for frequencies $\leqslant 0.1 \times 10^{14} \mathrm{rad} \mathrm{s}^{-1}$. Indeed, with the package QuTiP, the stationary solutions obtained numerically at these frequencies did not converge to the right solution. Correspondingly, the obtained efficiency is a very unstable function of the frequency. To overcome this problem, we solved the master equation using the software MATHEMATICA, imposing explicitly the use of a given number of accuracy digits. This number increases with decreasing frequencies and reaches 100 at $\omega=0.00001 \times 10^{14} \mathrm{rad} \mathrm{s}^{-1}$. Note that for frequencies $\omega \geqslant 0.1 \times 10^{14} \mathrm{rad} \mathrm{s}^{-1}$, both QuTiP and MATHEMATICA give the same results.

[29] R. L. Haupt and S. E. Haupt, Practical Genetic Algorithms (Wiley, Hoboken, 2004).

[30] M. Saffman, T. G. Walker, and K. Mølmer, Rev. Mod. Phys. 82, 2313 (2010).

[31] S. Komiyama, O. Astafiev, V. Antonov, T. Kutsuwa, and H. Hirai, Nature (London) 403, 405 (2000).

[32] C. Kammerer, S. Sauvage, G. Fishman, P. Boucaud, G. Patriarche, and A. Lemaître, Appl. Phys. Lett. 87, 173113 (2005).

[33] E. Homeyer, J. Houel, X. Checoury, F. Delgehier, S. Sauvage, P. Boucaud, R. Braive, L. Le Gratiet, L. Leroy, A. Miard, A. Lemaître, and I. Sagnes, Appl. Phys. Lett. 95, 041108 (2009).

[34] A. Barreiro, H. S. J. van der Zant, and L. M. K. Vandersypen, Nano Lett. 12, 6096 (2012).

[35] D. Wasserman, T. Ribaudo, S. A. Lyon, S. K. Lyo, and E. A. Shaner, Appl. Phys. Lett. 94, 061101 (2009). 